\title{
La interrupción y no publicación de los ensayos clínicos es un hecho frecuente que debe evitarse
}

\section{Discontinuation and non-publication of clinical trials is a common fact that should be avoided}

Kasenda B. JAMA. 2014;311(10):1045-1051.

\section{Objetivos}

Determinar la prevalencia, características y estado de publicación de los ensayos clínicos aleatorizados (ECA) interrumpidos. Investigar los factores asociados con la interrupción y no publicación de los estudios.

\section{Diseño y lugar}

Cohorte retrospectiva basada en el seguimiento de protocolos presentados entre los años 2000 a 2003 en seis comités de ética en investigación (CEI) de Suiza, Alemania y Canadá.

\section{Medición de resultados principales}

Se registraron las características de los ECA. Se determinó el estado de finalización del protocolo, las causas de discontinuación y estado de publicación a abril de 2013, determinado por la correspondencia con los CEI, una búsqueda bibliográfi- ca y una encuesta realizada a los investigadores principales.

\section{Resultados principales}

En el periodo analizado se presentaron 3.819 protocolos en los CEI participantes. Se incluyeron en el análisis 1.017 protocolos correspondientes a ECA: 894 incluían pacientes y 123 voluntarios sanos. Después de una mediana de seguimiento de 11,6 años (rango: 8,8 a 12,6 años) se detectó que 253 de 1.017 ensayos presentados fueron discontinuados (24\%; IC95\% 22,3 a 27,6\%). Los ECA que involucraban pacientes fueron interrumpidos con mayor frecuencia que aquellos que involucraban voluntarios sanos $(27,9 \%$ vs 3,3\%). La discontinuación de los ensayos fue informada a los CEI en sólo 96 de 253 casos (37,9\%; 32 a 44,3\%). La principal causa de discontinuación fue escaso reclutamiento de pacientes $(9,9 \% ; 8,2$ a $12 \%)$ (ver tabla 1$)$. La tabla 2 resume los principales factores asociados a la no publicación de ECA.

Tabla 1: Principales factores asociados a la discontinuación de ensayos clínicos aleatorizados (ECA) debido a pobre reclutamiento en los ECA que involucraron pacientes.

\begin{tabular}{|c|c|c|c|c|}
\hline \multirow[b]{2}{*}{ Caracteristicas } & \multicolumn{2}{|c|}{ ECA, №. (\%) } & \multicolumn{2}{|c|}{ Análisis multivariable } \\
\hline & $\begin{array}{l}\text { Suspendido por pobre } \\
\text { reclutamiento }(\mathrm{n}=90)\end{array}$ & $\begin{array}{c}\text { Completado } \\
(n=526)\end{array}$ & OR (95\% Cl) & $\mathbf{P}$ \\
\hline Tamaño muestral planificado, mediana (RIC) & $180(80 \text { a } 320)^{a}$ & $368(154 \text { a } 800)^{a}$ & $0,96(0,92 \text { a } 1,00)^{\alpha, \varepsilon}$ & 0,04 \\
\hline Financiado por la industria (vs. investigador), $n$ & $34(37,8)$ & $371(70,5)$ & $0,25(0,15$ a 0,43$)$ & $<0,001$ \\
\hline
\end{tabular}

RIC: rango Intercuartilo, a: Se excluyeron ECA con datos faltantes para cálculo de tamaño muestral, £. En incrementos de a 100.

Tabla 2: Factores asociados con la no publicación de ensayos clínicos aleatorizados (ECA).

\begin{tabular}{|c|c|c|c|c|}
\hline \multirow[b]{2}{*}{ Garacteristicas } & \multicolumn{2}{|c|}{ ECA, №. (\%) } & \multicolumn{2}{|c|}{ Análisis multivariable } \\
\hline & $\begin{array}{c}\text { No Publicado } \\
(n=451)\end{array}$ & $\begin{array}{c}\text { Publicado } \\
(n=566)\end{array}$ & OR $(95 \%$ Cl) & $\mathbf{P}$ \\
\hline Tamaño muestral planificado, mediana (RIC) & $120(40 \text { a } 330)^{a}$ & $303(100 \text { a } 745)^{\alpha}$ & $0,95(0,92 \text { a } 0,97)^{\varepsilon}$ & $<0,001$ \\
\hline Multicéntrico (vs centro único) & $280(62,4)$ & $470(83,0)$ & $0,50(0,32$ a 0,76$)$ & 0,001 \\
\hline Financiado por la industria (vs investigador) & $279(61,9)$ & $358(63,3)$ & $1,68(1,20$ a 2,34$)$ & 0,002 \\
\hline Discontinuado (vs completado) & $140(37,6)^{\epsilon}$ & $114(20,1)^{\epsilon}$ & $3,19(2,29$ a 4,43$)$ & $<0,001$ \\
\hline ECA con pacientes (vs voluntarios sanos) & $364(80,7)$ & $530(93,6)$ & $0,36(0,20$ a 0,63$)$ & $<0,001$ \\
\hline
\end{tabular}

RIC: rango Intercuartilo, a: Se excluyeron ECA con datos faltantes para cálculo de tamaño muestral, £. En incrementos de a 100 . €: Se excluyeron ECA sin certezas de que fueron completados.

\section{Conclusiones}

En la muestra analizada de protocolos, la interrupción de los ECA fue frecuente, siendo la principal razón de discontinuación el insuficiente reclutamiento de pacientes. Debería asegurarse la comuni- cación a los CEI de la suspensión de los estudios así como la publicación de sus resultados.

Fuente de financiamiento: Swiss National Science Foundation y German Research Foundation. 


\section{Comentario}

Los ensayos clínicos de adecuada calidad científica constituyen la principal fuente de evidencia acerca de la seguridad y eficacia de las intervenciones médicas. Su desarrollo implica un elevado consumo de recursos. Sin embargo, la interrupción y falta de publicación de estos estudios es un hecho frecuente que ha sido descripto en las distintas especialidades médicas ${ }^{1,2}$. A pesar de las implicancias éticas que conlleva la suspensión de un ECA hay escasa literatura que haya investigado las características y publicación de los ensayos discontinuados. En el presente trabajo, el seguimiento de los estudios mostro que un $25 \%$ de los ensayos clínicos no fueron finalizados. La causa más frecuente fue el escaso reclutamiento de pacientes. Los autores encontraron que la presencia de financiamiento por la industria y el mayor tamaño muestral estimado eran "factores protectores" de discontinuación por escaso reclutamiento. Una adecuada planificación de los ensayos y la realización de estudios piloto permitirían evaluar con anticipación la factibilidad de alcanzar un tamaño muestral suficiente para demostrar los objetivos propuestos, evitando el gasto de recursos innecesarios y someter a los participantes a riesgos sin alcanzar la meta del estudio. Por otro lado los autores encontraron que sólo un $56 \%$ de los estudios fueron publicados. La interrupción del ensayo fue el factor de riesgo que presento mayor asociación con la no publicación, seguido por el patrocinio del estudio por la industria. Considerando que las decisiones clínicas deberían sustentarse en la literatura publicada es necesario que todos los datos de aceptable calidad se encuentren disponibles. La publicación selectiva de los estudios clínicos tiene como resultado el "sesgo de publicación" que podría conducir a la toma de decisiones erróneas. A pesar de ello, la ausencia de publicación o publicación parcial es un hecho frecuente ${ }^{3}$. Su prevención es importante para asegurar la total diseminación del conocimiento y también para permitir combinar la totalidad de los resultados en meta-análisis.

\section{Conclusiones del comentador}

Considerando la escasez de presupuesto para investigación, es necesario desarrollar estrategias que aumenten su eficiencia evitando la discontinuación de los ensayos clínicos por inadecuado reclutamiento. Al mismo tiempo estimular la publicación de los resultados de los ECA permitirá aumentar la transparencia en la investigación clínica.

María del Pilar Arias López [ Hospital de Niños Dr. Ricardo Gutiérrez. Unidad de Cuidados Intensivos Pediátricos. mpariaslopez@gmail.com ]

Arias Lopez MP. La interrupción y no publicación de los ensayos clínicos es un hecho frecuente que debe evitarse. Evid Act Pract Ambul. 2015;18(2):44-45.Abr-Jun. Comentado de: Kasenda B, von Elm E, You J y col. Prevalence, characteristics, and publication of discontinued randomized trials. JAMA. 2014; 311(10):1045-1051. PMID: 24618966.

\section{Referencias}

1. Rosenthal R, y col. Completion and Publication Rates of Randomized Controlled Trials in Surgery: An Empirical Study. Ann Surg 2014:00:1-6.

2. Chapman Stephen J, y col. Discontinuation and nonpublication of surgical randomised controlled trials: observational study BMJ 2014;349:g6870.

3. Malički M, von Elm,E, Marušić A. Study Design, Publication Outcome, and Funding of Research Presented at International Congresses on Peer Review and Biomedical Publication. JAMA 2014; 311(10):1065-1067.

\begin{abstract}
Q ertenecer significa dar relevancia a nuestro trabajo cotidiano, reunirnos con colegas para compartir nuestras ideas, investigar y difundir nuestros conocimientos con otros profesionales. Asi como asesorar al gobierno y a otras instituciones privadas en la confección de guias que implican cambios en las politicas de Salud Pública, como queda demostrado con la participación de miembros de nuestra asociación en la confección de la Guia Nacional para la EPOC y la revisión del certificado de aptitud fisica.

Además significa integrarnos como médicos de familia a otras asociaciones de Medicina Familiar a nivel nacional, y compartir nuestro saber a nivel internacional, difundiendo nuestra práctica en Congresos dentro y fuera del pais.
\end{abstract}

¡Vos también podes formar parte de esta transformación!

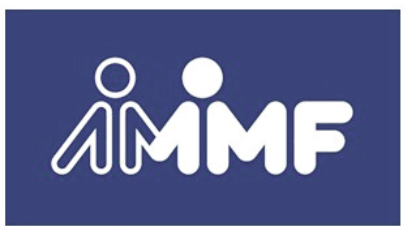

Asociación Metropolitana de Medicina Familiar.

Si queres asociarte o que te brindemos mayor información: secretaria.ammf@gmail.con

Seguinos en Facebook: www.facebook.com/AsociacionMetropolitanadeMedicinaFamiliar 